\title{
Hume and the Argument for Biological Design
}

\author{
by Graham Oppy
}

Here are three characteristic recent passages on the subject of Hume and the argument for design:

Many philosophers now regard David Hume’s Dialogues Concerning Natural Religion (1779) as the watershed in this argument's career. Before Hume, it was possible for serious people to be persuaded by the argument, but after the onslaught of Hume's corrosive skepticism, the argument was in a shambles and remained that way forever after. Biologists with an interest in the history of this idea often take a different view (Dawkins, 1986), seeing the publication of Darwin's Origin of Species as the watershed event. For the first time, a plausible non-theistic explanation of adaptation was on the table. ... It is possible to pose the question about the history of the design argument in two ways. The first is sociological: When (if ever) did educated opinion turn against the design argument? With respect to this question, it is quite clear that Hume's Dialogues did not put a stop to the argument. ... However, this sociological fact leaves unanswered the second historical question we can ask about the design argument. When (if ever) was the argument shown to be fatally flawed? Many philosophers nowadays think that Hume dealt the deathblow. ... [Discussion of two Humean objections excised.] ... Hume produced other criticisms of the design argument, but these fare no better than the two I have described here. Part of the problem is that Hume has no serious alternative 
explanation of the phenomena he discusses. It is not impossible that the design argument should be refutable without anything being provided to stand in its stead. For example, this could happen if the hypothesis of an intelligent designer were incoherent or self-contradictory. But I see no such defect in the argument. It does not surprise me that intelligent people strongly favoured the design hypothesis when the only alternative available to them was random physical processes. But Darwin entirely altered the dialectical landscape of this problem. His hypothesis of evolution by natural selection is a third possibility; it requires no intelligent design, nor is natural selection properly viewed as a “random physical process”. (Sober (1993:30-6))

I feel more in common with the Reverend William Paley than I do with the distinguished modern philosopher, a well-known atheist, with whom I once discussed the matter at dinner. I said that I could not imagine being an atheist at any time before 1859, when Darwin’s Origin of Species was published. 'What about Hume?', replied the philosopher. 'How did Hume explain the organised complexity of the living world?', I asked. 'He didn’t', said the philosopher. 'Why does it need any special explanation?'. Paley knew that it needed a special explanation; Darwin knew it, and I suspect that in his heart of hearts my philosopher companion knew it too. ... As for David Hume himself, it is sometimes said that that great Scottish philosopher disposed of the Argument from Design a century before Darwin. But what Hume did was criticise the logic of using apparent design in nature as positive evidence for the existence of a God. He did not offer any alternative explanation for apparent design, but left the question open. An atheist before Darwin could have said, following Hume: 'I have no explanation for complex biological design. All I know is that God isn’t a good explanation, so we must wait and hope that somebody comes up with a better one'. I can't help feeling that such a position, though logically sound, would have left one 
feeling pretty unsatisfied, and that although atheism might have been logically tenable before Darwin, Darwin made it possible to be an intellectually fulfilled atheist. I like to think that Hume would agree, but some of his writings suggest that he underestimated the complexity and beauty of biological design. (Dawkins (1986:5-6))

It may be argued that there is still something that calls for further explanation. ... There is only one actual universe, with a unique set of basic materials and physical constants, and it is therefore surprising that the elements of this unique set-up are just right for life when they might easily have been wrong. I suspect, however, that this objection also is being presented in a question-begging way. Though some small variation from the actual initial materials and constants would, perhaps, eliminate the possibility of life's having developed as it did, we really have no idea of what other interesting possibilities might have been latent within others of the endless range of possible initial conditions. We are not in a position, therefore, to regard the actual initial materials and constants as a uniquely fruitful set, and as surprising and as specially calling for further explanation on that account. Once these matters are cleared up, we can see that the shift of topic due to the work of Darwin and his successors greatly diminishes the plausibility of the argument for design. The reciprocal adjustments of structures and functions in myriads of different organisms are indeed so delicate and complicated as to be initially surprising in the extreme, and not merely to invite but to require a search for some further explanation; and then the hypothesis of design is at least one to be considered among others. But we find nothing comparable to this in sub-atomic particles or the laws that govern them. Atomic and nuclear physics are, no doubt, intricate enough to be of theoretical as well as practical interest, but we cannot see them as involving reciprocal adjustments which might plausibly be taken as signs of purposiveness. (Mackie (1982:141-2)) 
The views expressed in these passages -- and the examples could easily be multiplied -- seem to me to be almost entirely wrong. Sober claims: (i) that Hume produces no good criticisms of design arguments; (ii) that Hume offers no serious alternative explanations of (biological) design; and (iii) that design was the best explanation available prior to 1859. Dawkins claims: (i) that one could not have been an intellectually fulfilled atheist prior to 1859; (ii) that Hume offered no alternative explanations of (biological) design; and (iii) that Paley knew that there must be some non-Humean explanation of apparent biological design. Mackie claims: (i) that the work of Darwin greatly diminishes the plausibility of design arguments; and (ii) that there are no reciprocal adjustments in cosmology which are as plausible candidates for evidence of design as cases from biology. None of these claims can withstand scrutiny, as I shall now attempt to demonstrate. More exactly, I shall argue: (a) that the claim that Hume offered no (serious) alternative explanations of apparent (biological) design is, perhaps, strictly correct, but that, taken alone, it misrepresents the strength of the resources which Hume provided for developing (serious) alternative explanations of apparent (biological) design; and (b) that all of the other claims are simply false.

Arguments for design proceed from certain features of the world -- complexity of organisation, complexity of structure, complexity of adaptation -- to a conclusion which entails that those features are evidence that the world is a product of intentional design. The features in question can belong to cosmology, or particle physics, or chemistry, or biochemistry, or biology, or any of a number of other disciplines. The eighteenth and 
nineteenth century versions of these arguments focussed heavily -- though not exclusively -on: (i) the physical structure of the solar system; (ii) the structural organisation of (parts of) plants and animals; and (iii) the providential arrangement of conditions on the surface of the earth. Often, arguments were restricted to examples drawn from biology, (presumably) because it was felt that an overwhelming case could be constructed by considering these alone; but there are numerous examples -- for instance, among the Boyle lectures and Bridgewater Treatises -- of arguments which are developed entirely from non-biological cases. ${ }^{1}$ Also, while the conclusion of the argument might have been taken merely to be that there is at least one designer, the characteristic conclusion of the eigtheenth and nineteenth century design arguments was that there is a unique, very good, very powerful, very wise designer (or even, that there is a unique, omnibenevolent, omnipotent, omniscient designer).

The interpretation of Hume's Dialogues is extremely controversial. Some scholars think that Hume intended to defend a deistic explanation of apparent design. Others think that he used the dialogue format to disguise the force of the best case which could be made against any hypothesis of design. And there are other positions which might be taken as well. Whatever the truth of the matter, it is clear that one can choose from the materials and techniques which Hume offers to construct a powerful case against any hypothesis of design -- i.e. it is possible to read the Dialogues as a sourcebook for vigorous attacks on the eighteenth and nineteenth century design arguments.

Among the powerful objections which an atheist or agnostic (or even, at least for the first two objections, deist) might take from the Dialogues, there are at least the following three: (1) It is obviously hopeless to think that the inference to a very good, very powerful and very wise designer is a good one, given just the evidence of apparent design: for there are lots of evils in the world, lots of imperfect adaptations of structure to function, lots of wasted materials, and 
so on. (Dialogues, Parts X and XI: “... the inaccurate workmanship of all the springs and principles of the great machine of nature”). (2) Not unrelatedly, there are many alternative hypotheses which account for the evidence of apparent design at least as well as the hypothesis that there is a unique, very good, very powerful, very wise designer: for example, that there is a committee of designers, who couldn't come to unanimous agreement on all matters (hence the imperfections in the final product); that there is a fairly incompetent but very persistent designer, who botches up universe after universe; and so on. (Dialogues, Parts V-VIII.) (3) Even if we restrict ourselves to the conclusion that there is at least one (more or less) intelligent designer, we face the problem of explanatory advance: given that we want to explain the occurrence of certain kinds of complexity in the world, what advantage do we gain by postulating similar kinds of unexplained complexity in some other realm? If we are content to leave the complex intentions, etc. of the designer(s) unexplained, why shouldn’t we have been content to leave the original complexity in the world unexplained? And how could we even pretend to an explanation of those complex intentions, etc? (Dialogues, Part IV, especially pp.34-35.)

The examples from the last paragraph serve to refute the first of Sober's claims. Hume produced lots of good criticisms of arguments for design. For, first, he produced compelling criticisms of the typical eighteenth and nineteenth century inference to the existence of a unique, very good, very wise, and very powerful designer. And, second, he produced at least one very powerful criticism of the more modest inference to the existence of at least one (more or less) intelligent designer. Note that I don’t say that this second objection is decisive: there are hard questions about theoretical virtues and explanatory advance still to be addressed. However, I do think that it is clear that this is a very powerful criticism. (There is some irony in the fact that Dawkins twice makes use of this second Humean criticism. Consider: “[O]f course any God capable of intelligently designing something as complex as 
the DNA/protein replicating machine must have been at least as complex and organised as the machine itself. ... To explain the origin of the DNA/protein machine by invoking a supernatural Designer is to explain precisely nothing, for it leaves unexplained the origin of the Designer. You have to say something like 'God was always there', and if you allow yourself that kind of lazy way out, you might as well just say 'DNA was always there' or 'Life was always there', and be done with it.” (Dawkins (1986:141)) And: “If we want to postulate a deity capable of engineering all the organised complexity in the world, either instantaneously or by guiding evolution, that deity must already have been vastly complex in the first place. The creationist, whether a naive Bible-thumper or an educated bishop, simply postulates an already existing being of prodigious intelligence and complexity. If we are going to allow ourselves the luxury of postulating organised complexity without offering an explanation, we might as well make a job of it and simply postulate the existence of life as we know it!” (Dawkins (1986:316)).)

Of course, there are still some subtleties which need to be addressed here. Hume's objection (as I am construing it) is to the inference from apparent design, and not necessarily to the idea that the apparent design could be explained by the existence of a designer. If one supposes that one has independent reasons for holding that there is a very intelligent creator -- for example, on the basis of an ontological argument -- then one will quite rightly think that "Hume’s objection” is not very compelling. But that isn't how the dialectic goes: the eighteenth and nineteenth century proponents of the design arguments took those arguments to be the best (perhaps even the only) basis for an inference to the existence of God. I claim that Hume's objections to these people were very strong indeed. 
Despite the claims of Sober and Dawkins to the contrary, it seems to me that Hume did more than merely criticise the inference from apparent design, i.e. it seems to me that he offered alternative explanations of the apparent evidence for design, and moreover that he offered serious alternative explanations of this apparent evidence. Perhaps I am wrong about this; but, in any case, all I really need to establish is that Hume could have offered alternative explanations of the apparent evidence for design, i.e. that he had available to him serious alternative explanations of this apparent evidence. If merely Humean alternative explanations are serious competitors to the design hypothesis, then that it enough to defeat the claims of Sober and Dawkins about the logical importance of the publication of The Origin of Species (There are at least four different questions here: (i) What alternative explanations are explicitly offered by Hume? (ii) What alternative explanations are implicitly developed by Hume? (iii) What alternative explanations are natural extensions or analogues of explanations which are developed by Hume? (iv) What alternative explanations were available to Hume, given the state of scientific knowledge at the time? I shall -- more or less -- grant to Sober and Dawkins that Hume does not explicitly offer serious alternative explanations to design. But this concession leaves open the question of the logical importance of Humean considerations for the pre-1859 argument for design.)

Before I try to defend these claims, there are two important preliminary points to note. First, we need to remind ourselves of what Hume (and Paley) didn't know. As Dawkins and Sober emphasise, they knew nothing about the degree of complexity of organisms and (the mechanism of) natural selection. But they also knew nothing (much) about: geology (the age of the earth); thermodynamics (the second law, which concerns the overwhelming statistical likelihood of increases in entropy in isolated systems); the expanding universe (the Hubble 
shift); and the Big Bang (the cosmic microwave background radiation, the COBE data, the age of the universe). Second, we need to remind ourselves of what Hume (and Paley) took themselves to know (or, at least, could have reasonably allowed themselves to believe, if they were well-versed in current scientific ideas). According to the then current Newtonian cosmology, the universe is both spatially and temporally infinite. At any time, it contains infinitely many stars -- and, hence, presumably, infinitely many solar systems. (The inference to infinitely many solar systems would probably have been rejected by Newton himself, on the grounds that God must constantly intervene to preserve the stability of our solar system. However, many subsequent Newtonians did not accept this part of Newton’s views. In Part VIII of the Dialogues, Philo develops some alternative explanations of apparent design based on the assumption that the universe contains only finitely many particles of matter. These explanations are in conflict with the best physics of the day.)

How, then, did (or could) Hume seek to explain the apparent evidence for design? I suggest that he had (or could have had) three strategies, all with a reasonable degree of plausibility.

(1) First, one might offer to explain apparent present design in terms of apparent past design: at any time, the order in the world is explained as the product of order which existed at an even earlier time. Given ignorance about the finitude of the past and the second law of thermodynamics, this explanation doesn't seem utterly implausible. (Hume does defend explanatory regresses (Part IX); and he does note, several times, that present apparent design can be explained in terms of immediately past apparent design (e.g., Part III). However, he doesn’t actually undertake to explain present apparent design in terms of an infinite regress -i.e. this explanation is merely Humean. On the other hand, he does offer to explain present order in terms of an "eternal inherent principle of order” -- Part VI -- and this explanation could perhaps be expanded in the way I have suggested.) 
(2) Second, one might offer to explain apparent design in terms of an ensemble of worlds: even though it is highly unlikely that apparent design could have arisen by chance, if there are sufficiently many different venues, then apparent design is bound to appear in lots of them. Against the Newtonian background, this looks like a pretty good explanation. (Hume actually gives a temporal version of this argument (Part VIII): if there are only finitely many possible states of the world, then in an infinite amount of time, each will appear infinitely often; and even if there are infinitely many possible states of the world, in an infinite amount of time, highly ordered members amongst the infinitely many possible states of the world are bound to appear. (A tacit assumption which appears to be at work here is that disordered states give rise to later states in a random fashion.) Hume also mentions various hypotheses which involve world ensembles -- e.g. in Part V (“Many worlds might have been bungled or botched”, p.39).)

(3) Third, one might offer to explain apparent design in terms of chance. Sure, it seems unlikely that the apparent design which we see arose by chance -- but it isn’t impossible, and there is no better explanation to be had. Consider a universe in which there is a galaxy with 10 billion stars with planets each inhabited by 10 billion people. At a given time t, each person rolls a fair 100-sided die, and logs the result on a central computer. By an astonishing chance -- one in $100^{10^{20}}$-- every one of the $10^{20}$ people records the number 17 . In this situation, in order to get the right answer -- namely, that it was just a gigantic fluke -- the people must refrain from entertaining supernatural explanations, etc. Moreover, once they have exercised the due methodological care -- ruling out collusion, etc. -- it seems to me that they should come to the conclusion that it was (probably) just a fluke. (More about this later.) To make the case more compelling, we should add something like the following details to the story: There is only ever one trial. The dice are manufactured, issued, recollected and destroyed by 
local authorities. Prior to distribution, and subsequent to recollection, the dice are subject to stringent tests -- including trials of a statistically significant sample -- which uncover no reason to think that the dice are individually or collectively biassed. These tests are witnessed -- perhaps even conducted -- by all who participate in the experiment. Moreover, there is no evidence for -- nor even rumours about -- any attempts to subvert the experiment. And the computer program, data entry, etc. are subject to equally stringent controls. Etc. (This suggestion may not sound Humean: Philo says (Part VI) that "Chance has no place, on any hypothesis”. However, Philo also says here that he prefers the hypothesis of an "eternal inherent principle of order in the world”, and this sounds suspiciously like an occult property of the kind lampooned in Part IV. Moreover, the context strongly suggests that Philo would prefer to say that order is just a brute fact, with no further explanation. ("Were I obliged to defend any particular system of this nature ..”) Perhaps there is room for debate about whether this should count as an "explanation"; however, the important point is that the hypothesis of chance (brute fact, no further explanation) is a perfectly respectable competitor, which needs to be taken into account.)

In my view, the considerations raised in the last three paragraphs show, contra Sober and Dawkins, that Hume did have (or could have had) serious alternative explanations of apparent design available, and moreover these considerations also show that design was not the best explanation prior to 1859. Of course, an atheist or agnostic prior to 1859 ought to have confessed that he didn’t know which was the correct explanation of apparent (biological) design. However, the problem should have appeared to be one of embarassment of riches: there are too many roughly equally good explanations, and no obvious means of choosing between them. (If Dawkins supposes that an intellectually fulfilled atheist needs to know everything, then it is plain that there never have been, and never will be, intellectually fulfilled atheists. Furthermore, given that the alternative hypotheses are plainly no worse than 
the hypothesis of design, it is also clear that parity of argument will entail that there could have been no intellectually fulfilled theists prior to 1859.)

Moreover, these same considerations show, contra Dawkins, that Paley did not know that there must be some other special explanation of apparent biological design. The problem, prior to 1859, was that no one had hit upon the correct explanation of apparent biological design, viz. the Darwinian explanation, which is manifestly vastly superior to any of the alternatives. However, prior to 1859 -- or, at least, prior to Darwin’s precursors -- no-one had even the faintest idea that there was such an explanation to be had. Of course, ex post facto, we can see that their situation was manifestly unsatisfactory -- there was a much better explanation to be had, if only they could find it. But what reason is there to suppose that they had any awareness of the existence of this better explanation? Often, we can't guess at the form which a good explanation will take until we have it; the case of apparent biological design is, in my view, simply a case in point.

There is a clear tension in the remarks which Dawkins makes about Hume. On the one hand, he claims -- on the basis of characteristically Humean reasoning -- that the theistic hypothesis isn't even a competitor, let alone a serious competitor, to the hypothesis of natural selection. On the other hand, he claims that, prior to 1859, atheists incurred special liabilities from their failure to hit upon a good explanation for apparent biological design. These two claims could only be reconciled by an argument which showed that the theistic hypothesis is greatly superior to the Humean alternatives -- but Dawkins nowhere attempts to produce such an argument. With good reason, in my view; I know of no persuasive argument for this conclusion.

Of course, there are (unpersuasive!) objections which might be offered against my claim that 
design was not obviously the best explanation prior to 1859. First, it may be said that a commitment to one complex system which arises by chance is better than a commitment to many complex systems which arise by chance -- better one big mystery than many little mysteries. However, it seems to me that this objection relies upon unfair methods of accounting. There is just one universe, with a level of complexity which is at least equalled by the level of complexity of its designer(s). The universe has many parts which are complex -but, corresponding to these parts, there are parts of the designer(s) which have a corresponding degree of complexity (the particular creative intentions which apply to those parts). To compare one unexplained complex system (the designer(s)) with many unexplained complex systems (the complex parts of the universe) is simply to cheat. Second, it might be said that more argument is required to show that the theistic hypothesis is not better than the Humean alternatives. I suppose that the Humean point about explanatory advance -- perhaps together with more general considerations about theoretical virtues -- constitutes a very powerful prima facie case for the claim that the theistic hypothesis is no better than the Humean competitors as an explanation of apparent design. At the very least, the examples show that it is very far from obvious that design was the best explanation of apparent design prior to 1859. (Perhaps, owing to the vagaries of history, prior probabilities were so distributed before 1859 that there is a sense in which design was the best overall explanation prior to 1859. However, even if it were so, this sociological fact would be irrelevant to our investigation of the logical status of the hypothesis of design. Naturalistic explanations were especially virtuous even then.) At least pro tem, then, I take it that the claim that design was not obviously the best explanation prior to 1859 is in pretty good shape. 
Even if I have managed to show that Sober and Dawkins underestimate the pre-1859 force of Humean objections to design arguments, I have not yet done anything much towards establishing the claim, contra Mackie, that Darwin’s work didn’t greatly diminish the plausibility of design arguments. Here, I can be brief. Apparent biological design is only one kind of apparent design. If there are other apparent kinds of design which seem to be about as much in need of explanation as apparent biological design, and which are untouched by Darwinian considerations, then the design argument as a whole is undamaged by the Darwinian assault. I claim that there are such cases: cosmology, for one. (I shall return to this topic in the next section.)

Why then did Darwin and his contemporaries suppose that the theory of evolution posed such a threat to the argument for design? I offer a sociological conjecture. For more than 150 years, many -- perhaps even most -- (intelligent) people had supposed that all the proof that one needed for the existence of a very good, very wise, very powerful designer could be found in apparent biological design. Moreover, the apparent design in question was such that anyone could appreciate it -- it didn’t require any background mathematical or physical knowledge, etc. -- and hence it could be readily appealed to from pulpit and editorial. As a result, the argument from biological design came to have a social importance which greatly exceeded its logical importance. Given that it was one of the common, shared assumptions of the day, that apparent biological design is the best, and all the needed, evidence for the existence of a very good, very wise, very powerful designer, it should not be surprising that Darwin's theory was greeted with considerable consternation. (It is perhaps worth noting that "the argument from the heavens” can’t proceed merely from a quick glance at the night sky -- the order alleged to be found there can only be discerned from lengthy astronomical observations (albeit observations which were once made solely by the unaided human eye). The "order” which so impressed the Greeks was principally an artefact of the rotation of the earth about its axis; but 
some reasonable data about the orbits of the planets could have been obtained by naked eye observation. Moreover, the arguments in the eighteenth and nineteenth century were able to draw upon telescopic data as well.)

Of course, the argument of the last paragraph is extremely hypothetical and simplistic. We have no figures to support the claims involved, nor any means of obtaining them. However, it is I think very plausible to hold that some suitably filled out sociological explanation of this form is correct. Contra Sober, it most certainly was not Darwin who dealt the fatal logical blow to design arguments -- though, plausibly, he and his successors did utterly demolish the argument for biological design.

Then again, even the claim that Darwin and his successors did utterly demolish the argument for biological design is not obviously correct. If we suppose that the world is deterministic -or, at least that, given a complete set of data for a single time and a complete set of data for infinity at all subsequent times, the laws determine all the data for all subsequent times -- then there is still the improbability of the initial data and conditions at infinity, which permit the later development of apparent evidence for biological design, to be accounted for. Obviously, there is no Darwinian explanation of this improbability in the offing -- so a Newtonian might well feel justified in maintaining that Darwin didn’t demolish all versions of the argument for biological design.

More generally, one might cast the current objection as follows: Darwin showed that the process of natural selection could have led to the gradual production of certain kinds of complexity from conditions in which those kinds of complexity were completely absent. Biological complexity -- complexity of function, structure and adaptation -- did not need to be produced in a single step, whether by chance or special creation. However, the Darwinian 
argument says nothing about the likelihood of obtaining initial conditions upon which natural selection can plausibly operate to produce biological complexity. Moreover, contra Mackie, there seems to be good reason to think that the likelihood of obtaining suitable conditions by chance is actually vanishingly small. At any rate, this is what the argument from cosmology -which turns out not to be entirely independent from the argument from biology -- tells us. (Mackie claims that, while life as it developed would have been impossible, we don't know what else might have developed. However, as we shall see, the "suitable conditions" in question are plausibly suitable conditions for anything which might plausibly be called "life”. Could there be anything which deserved to be called "life" in a universe which lasted for only a fraction of a second? Could there be anything which deserved to be called "life" in a universe which contained nothing but very dilute radiation? Setting aside very bizarre speculations, the answer seems to be: clearly not. At the very least, it is plain that it isn't just carbon-water-etc. based life which is at issue here.)

Inspired by the argument of the last two paragraphs -- to the correctness of which I make no commitment -- one might even suppose that there is a more or less a priori argument which entails that current improbability is bound to resist complete procedural explanation. Suppose that the current state of the world is improbable. Suppose further that there is a procedure which renders the present state of the world very highly probable given some range of past states of the world. Suppose that the procedure in question is instantiated. Then, the relevant range of past states of the world must be highly improbable -- for, if not, then the present state of the world would be highly probable, contrary to our initial assumption. (Of course, if the relevant range of past states is probable, then the conclusion to be drawn is that, despite appearances, the present state of the world is probable too. Perhaps it is this inference which defenders of the logical importance of cumulative selection for the argument for design have in mind. But it should be noted that there is nothing in the theory of cumulative selection 
which suggests that the relevant past states of the world are probable.)

The strongest current version of the argument for design comes from physics, in particular from cosmology. The general thread of these arguments is something like this: tiny variations in fundamental constants -- e.g. force strengths and particle masses -- at earlier stages of the universe would have led to the complete absence of nuclei, atoms, stars, and galaxies, and hence to the absence of anything which could deserve to be called life. Consequently, the existence of a universe in which there is life seems to be massively unlikely, and hence something which is in need of special explanation.

Many of the probabilities estimated in the "fine-tuning” arguments are extremely impressive. For example, Roger Penrose has calculated that -- in the absence of some new physical principle such as his "Weyl curvature hypothesis" -- there was just one chance in $10^{10^{123}}$ that a particular stage of the very early universe would have that smoothness required for the later development of life. Of course, Penrose's calculation requires assumptions which almost certainly are false; and, in general, it is very hard to estimate the robustness of the "finetuning” arguments. Cosmology -- and, in particular, that branch of cosmology which deals with the very early universe -- is an extremely controversial field, in which models rise and fall in rapid succession. (Indeed, some philosophers -- e.g. van Fraassen (1995) -- have gone so far as to suggest that "global cosmology" -- i.e. the attempt to provide physical models of "the universe" -- is not, properly speaking, a scientific pursuit. I think these philosophers are wrong, but I shan't attempt to argue the case here.) However, there is no reason to think that 
the very early universe fine-tuning arguments will just fade away. Moreover, there are other fine-tuning arguments -- e.g. those involving force-strengths and particle masses -- which have a much more firmly established theoretical basis. ${ }^{2}$

What should we say in the face of the apparent evidence for "fine-tuning”? Well, it seems to me that we are pretty much in the same position as Hume was in when he confronted the apparent evidence for biological design. (If some calculation like that of Penrose is correct, then the improbabilities involved might even turn out to be of roughly the same orders of magnitude.) Moreover, we seem to have roughly the same range of options:

(1) We can appeal to a regress: the reason why we have apparent "fine-tuning" at any time t is that we had apparent "fine-tuning" at earlier times. Even if the universe is temporally finite in the past, this argument might still be made to work provided that the universe is open -- i.e. provided that for any time, there is an earlier time. (This will be the case, for example, if time can be modelled by a segment of the real number line which is open at at least one end.) Of course, it may seem that there is something which still needs to be explained; namely, why the four (or more) dimensional universe exhibits apparent "fine-tuning”. One way to avoid this difficulty would be to adopt a three (or more) dimensionalist view. Perhaps there are other possibilities as well. It isn't obvious to me that this option is hopeless.

(2) We can appeal to an ensemble of "worlds". Perhaps there are lots of regions of spacetime with different values for the force-strengths, particle masses, initial smoothness, and so on. No matter how small the probabilities -- provided that they remain finite -- we only need a sufficiently large number of "worlds" to make the resulting outcomes more or less inevitable. (Perhaps there are infinitely many “worlds”; some recent cosmological theories allow this, and some do not.) Of course, it would be nice if we got the ensemble of "worlds" from 
physical theory -- and perhaps we will. (Some "inflationary” models of the early universe provide “worlds” which accomodate Penrose’s “fine-tuning” for smoothness, though they have other problems of their own.) Even if we don’t get an ensemble of “worlds” from physics, it isn't clear that this option is untenable.

(3) We can appeal to chance. Yes, there is some sense in which the "fine-tuning" arguments show that our universe is very unlikely -- but very unlikely things do happen. Once we’ve eliminated all of the methodologically respectable ways of trying to explain the apparent “fine-tuning”, we may be required to say that it is just a matter of chance. Or, if you prefer: that there simply is no explanation, but only a brute matter of fact. There is nothing evidently unrespectable about this option: from whence comes the idea that everything must have an explanation (which is both satisfying and accessible to us)? (A limited survey suggests a sharp division of opinion here (but not one which coincides with the theist/non-theist divide). Some people hold that it is just obvious that "fine-tuning" demands an explanation other than mere chance (at least if the numbers hold up). Others hold that it is equally obvious that "finetuning” does not demand an explanation other than chance (if the numbers hold up); there may be an explanation, but equally, there may not. Of course, these are ontological theses: they say nothing about the reasonableness of searching for an explanation, nor anything about the strength with which one might reasonably hold the belief that there is no further explanation.)

(4) We can appeal to design. Of course, if we take this option, we must provide an answer to the Humean objections to arguments for design -- but perhaps one might be able to reasonably believe that this can be done.

My list of options may very well be incomplete. (One deliberate omission is Leslie’s neo- 
Platonic hypothesis of a creatively effective ethical requirement. Despite Leslie’s disclaimers, it seems to me that this hypothesis requires commitment to an untenable theory of values; however, a proper argument in defence of my position would require an uncomfortably large digression from the main topics of this paper. I hope to discuss Leslie's positive views elsewhere.) There may be an option which is a better explanation of apparent "fine-tuning" to much the same kind of extent that cumulative selection is a better explanation of apparent biological design. But I can’t imagine what kind of explanation that would be. And so -- I claim -- I find myself in much the same kind of situation that Hume found himself in when he considered the argument for design (and, in particular, when he considered the argument for biological design). I don’t know whether apparent “fine-tuning” has an explanation. I don’t know whether one of the putative explanations which I have offered provides a correct explanation. I don't know whether there is an obviously better explanation which I have been unable to construct. But $I$ think that it is quite clear that the hypothesis of design does not provide the best explanation amongst those that I have been able to construct -- and that is enough to allow me to be a reasonably contented agnostic or atheist. Moreover, it is highly plausible to think that no story about cumulative selection will have any role to play in explaining the apparent "fine-tuning" -- and hence it is highly plausible to think that Darwinian considerations won't play any role in my rejection of this version of the argument for design. (One way in which my situation differs from Hume's is that, while it seems possible that future developments in physical theory might remove all of the apparent evidence for "fine-tuning", the apparent evidence for biological design is obvious for all to see. If the apparent evidence for "fine-tuning” did disappear, then some further re-assessment of design arguments might be required. However, it seems unlikely that the evidence for apparent fine-tuning will disappear. (For instance: some early states of the universe are massively improbable on purely thermodynamic grounds -- and yet without such massively improbable early states, there would almost certainly be no atoms, stars, etc. How is the 
extraordinarily low entropy of those early states to be explained?))

Even though I have rejected much of what Dawkins, Sober and Mackie say about the importance of Darwinian considerations for the argument for design, I should perhaps emphasise in closing that this implies no further disagreement with them about the importance of Darwin's achievement. As Dawkins says, Darwin could have showed Hume a thing or two -- in particular, he could have showed him the correct explanation of apparent biological design. Moreover, the philosopher who asked Dawkins why the organised complexity of the living world needed special explanation could also have learned something from Darwin: one obvious reason why it needs a special explanation is that, as a matter of fact, it has one! (This is another point at which the gulf between what we (collectively) know, and what Hume (and his contmporaries) knew, becomes important. Given knowledge of the immense age of the earth, the fairly complete fossil record, the structure and function of DNA, and so on, it would not, I think, take too much inspiration to arrive at the theory of natural selection -- i.e. it might well be quite obvious that there is a good explanation to be had (and, moreover, what form it will take). But neither Hume nor Darwin had this knowledge; and this is partly what makes Darwin's achievement so spectacular. (Actually, it is hard to imagine modern biology without the theory of cumulative selection -- from taxonomy to genetics, its influence is everywhere. Thus, experts in biological sciences face an even greater imaginative task when they try to see the world from a pre-Darwinian perspective.))

Hume's error -- if he made one -- was not to underestimate the complexity and beauty of biological design, but rather to underestimate the abilities of people to come up with novel scientific theories to explain features of the world which previously looked as though they could not be scientifically explained. (Actually, I don't think that there is much evidence that Hume made this mistake, hence the qualification. Another error which is sometimes alleged 
by defenders of design arguments is that Hume is too quick in moving to a position which entails that no evidence could be evidence of design. Thus, we find Leslie asking: what if the words “MADE BY GOD” were found all over the earth (at regular intervals in granite’s crystal pattern, in chains of particles, in the binary code for the ratio of two fundamental forces, on the skins of animals, etc.)? Well, I don't know. If the world were otherwise nonmagical, then Hume’s competing explanations still seem pretty good. This isn't to deny that there could be a world which wore the evidence of supernatural design on its face -- but it is to insist that it would need to be VERY different from the world which we actually encounter. Naturalistic explanations are as a matter of fact so good that they can tolerate a lot of apparent coincidences, particularly when those apparent coincidences would otherwise undermine naturalistically impeccable explanations. (There's no independent evidence that English is the language of the designers -- rather than a language which evolved in the usual way on earth -or that there are no other intelligent creatures in the universe. So why should English be the language in which the designers leave signatures? Etc. If the message appeared to each in a language which s/he spoke, then the phenomena would be magical, i.e. not susceptible of naturalistic explanation -- but that would be a VERY different world to ours.)) ${ }^{3}$

\section{References}

Dawkins, R.: 1986, The Blind Watchmaker, Penguin, London.

van Fraassen, B.: 1995, ““World” Is Not A Count Noun’ Noûs , 29, 139-157.

Hume, D.: 1948, Dialogues Concerning Natural Religion (edited with an introduction by H. Aiken), Macmillan, London.

Leslie, J.: 1989, Universes , Routledge, London. 
Mackie, J.: 1982, The Miracle Of Theism, Oxford University Press, Oxford.

Sober, E.: 1993, The Philosophy Of Biology, Westview, Boulder, Colorado.

Notes

${ }^{1}$ William Derham's 1712 Boyle Lectures were published under the title Astro-Theology: Or,

A Demonstration Of The Being And Attributes Of God, From A Survey Of The Heavens.

William Whewell’s 1833 Bridgewater Treatise was titled Astronomy And General Physics

Considered With Reference To Natural Theology. My favourite title amongst the Bridgewater

Treatises is William Prout's 1834 Chemistry, Meteorology, And The Function Of Digestion.

But I digress.

${ }^{2}$ See Leslie (1989) for a forceful presentation of the then current evidence for apparent "finetuning”.

${ }^{3}$ Thanks to James Maclaurin, Karen Neander, Jack Smart, and an anonymous referee from Biology and Philosophy for critical discussion of, and helpful suggestions about, the material presented in this paper. 